\title{
Facilitating the Guangdong-Hong Kong-Macao Greater Bay Area's Participation in the Dual Circulation Economy
}

\author{
Han Yonghui ${ }^{1}$, Mai Jinghua ${ }^{1}$, Li Qing ${ }^{1 *}$, Zhang Fan $^{1}$, Luo Xiaofei ${ }^{1}$ \\ ${ }^{1}$ Guangdong institute of international strategy, Guangdong university of foreign studies, GDUFS, Guangzhou, China
}

\begin{abstract}
The Sino-US conflicts and the COVID-19 have significantly impacted the Chinese economy and the Chinese government has proposed the "Dual Circulation Economy" strategy as countermeasure. As one of the most developed regions in China, the Guangdong -Hong Kong-Macao Greater Bay Area is expected to shoulder responsibilities in this ambition. In this paper, we analyze the challenges for the Greater Bay Area to participate from the perspective of industrial development. We then employ a quantitative method to identify the industrial potential of the Greater Bay Area cities in facilitating the dual circulation economy. At last, we provide several policy recommendations including promoting cross-regional industrial cooperation and enhancing the spatial spillover effects of core cities.
\end{abstract}

\section{INTRODUCTION}

The global political and economic order is in turmoil. Especially under the impact of the COVID-19, the global economic governance system and the world market have been greatly overturned (Yang, 2020; Lin, 2020; Ferrannini et al., 2021; Bishop \& Payne, 2021) ${ }^{[1,2,3,4]}$. To address the turbulent, the Political Bureau of the Communist Party of China Central Committee points out that China still faces uncertainties that would extend to the medium and long run. It urged the country to construct a "dual circulation" economic pattern, in which domestic economic cycle plays a leading role while international economic cycle act as the extension and supplement of the domestic cycle. The Chinese economy is about to enter a new era. Under the pressure of the external environment, to lay a solid foundation for the construction of the "dual circulation" is of significance (Shen \& Zhao, 2020; Sheng, 2020). ${ }^{[5,6]}$ It yields extensive benefits to further the reform and opening-up, achieve high-quality economic development, contribute the Chinese wisdom to global governance, and facilitate the building of a worldwide community with a shared future for mankind (Blanchard \& Zeng, 2020). ${ }^{[7]}$ As one of the most vital region in China's economic development, the Guangdong-Hong KongMacao Greater Bay Area (Greater Bay Area) has the comparative advantage of connecting the domestic and foreign institutions, rules and markets (Eddie et al., 2020; Yan \& Liu, 2020). ${ }^{[8,9]}$ When the world is in structural changes, it is necessary to explore the role of the Greater Bay Area in participating in the "dual circulation".

In this paper, we analyze the challenges faced by the Greater Bay Area in participating in the "dual circulation", and then propose the policy recommendations accordingly.

\section{CHALLENGES}

\subsection{The spatial layout of industries}

The industrial structures are homogeneous. The manufacturing similarity coefficients of the nine inland cities in the Greater Bay Area are mostly over 0.7, among which the coefficients of Shenzhen-Dongguan-Huizhou and Foshan-Zhongshan-Zhuhai are over 0.8 (Table 1). While "industries with value-added ratio $\geq 5 \%$ " are defined as dominant industries, "industries with the dominant position in more than 5 cities" are defined as highly homogenized industries. Computer communication, electrical machinery, basic chemical and metal products are highly homogeneous industries within the Greater Bay Area. While the former two are the dominant industries in almost all the inland cities of the Greater Bay Area, accounting for a high proportion of industrial added value. If there is a lack of coordination and communication in the cities' industrial policy design, the phenomenon of industrial homogeneity will further deteriorate (Han et al., 2017). ${ }^{[10]}$ 
Table1. Similarity coefficient matrix of manufacturing structure in the Pearl River Delta region, 2018

\begin{tabular}{|c|c|c|c|c|c|c|c|c|c|}
\hline & $\begin{array}{c}\text { Guang } \\
\text { zhou }\end{array}$ & $\begin{array}{c}\text { Shen } \\
\text { zhen }\end{array}$ & $\begin{array}{c}\text { Fo } \\
\text { shan }\end{array}$ & $\begin{array}{c}\text { Dong } \\
\text { guan }\end{array}$ & $\begin{array}{c}\text { Hui } \\
\text { zhou }\end{array}$ & $\begin{array}{c}\text { Zhong } \\
\text { shan }\end{array}$ & $\begin{array}{c}\text { Jiang } \\
\text { men }\end{array}$ & $\begin{array}{c}\text { Zhu } \\
\text { hai }\end{array}$ & $\begin{array}{c}\text { Zhao } \\
\text { qing }\end{array}$ \\
\hline $\begin{array}{c}\text { Guang } \\
\text { zhou }\end{array}$ & 1.00 & -- & -- & -- & -- & - & -- & -- & - \\
\hline $\begin{array}{c}\text { Shen } \\
\text { zhen }\end{array}$ & 0.32 & 1.00 & -- & -- & -- & - & - & -- & - \\
\hline $\begin{array}{c}\text { Fo } \\
\text { shan }\end{array}$ & 0.43 & 0.26 & 1.00 & -- & -- & - & - & -- & - \\
\hline $\begin{array}{c}\text { Dong } \\
\text { guan }\end{array}$ & 0.39 & 0.95 & 0.46 & 1.00 & -- & - & - & -- & - \\
\hline $\begin{array}{c}\text { Hui } \\
\text { zhou }\end{array}$ & 0.45 & 0.89 & 0.37 & 0.88 & 1.00 & - & -- & -- & - \\
\hline $\begin{array}{c}\text { Zhong } \\
\text { shan }\end{array}$ & 0.50 & 0.63 & 0.88 & 0.78 & 0.69 & 1.00 & - & -- & - \\
\hline $\begin{array}{c}\text { Jiang } \\
\text { men }\end{array}$ & 0.50 & 0.38 & 0.77 & 0.57 & 0.49 & 0.77 & 1.00 & -- & - \\
\hline $\begin{array}{c}\text { Zhu } \\
\text { hai }\end{array}$ & 0.47 & 0.72 & 0.78 & 0.79 & 0.77 & 0.94 & 0.68 & 1.00 & - \\
\hline $\begin{array}{c}\text { Zhao } \\
\text { qing }\end{array}$ & 0.39 & 0.31 & 0.62 & 0.48 & 0.39 & 0.56 & 0.73 & 0.41 & 1.00 \\
\hline
\end{tabular}

The polarization effect is severe. In 2019, Shenzhen's R\&D investment accounted for 5\% of GDP, more than the sum of the rest of the Greater Bay Area cities. Its level of innovation investment has obvious advantages. In highend electronic information manufacturing industry, Shenzhen, Dongguan and Huizhou take advantage of their geographic advantages, leading to a "strong in the east while weak in the west" situation in the Greater Bay Area $(\mathrm{Xu} \& \mathrm{Wu}, 2019) .{ }^{[11]}$ In addition, Guangzhou and Shenzhen possess abundant industrial resources and favorable policies to attract high-end elements, and there is a huge gap in the factor resources available for deployment between the center and the peripheral cities. It is often difficult for the secondary cities in the Greater Bay Area to enjoy the dividends brought by the industrial division of labor. The polarization of industrial development is gradually exacerbating (Liu \& Liu, 2019). ${ }^{[12]}$

\subsection{The linkage of industrial development}

Top-level design is incomplete. The lack of forwardlooking and top-level design in some cities restricts the balanced layout of industries, leading to weak spatial spillover effects. The phenomenon of resource mismatching and blind positioning of advanced industries is another headache in the area (Li \& Deng, 2019). ${ }^{[13]}$ Meanwhile, the industrial platforms set up by the central government are mostly distributed in Guangzhou and Shenzhen. Local governments are often keen on supporting industries that are short-period and yield quick returns, ignoring the significance of those require long a long time. Moreover, the industrial platforms in peripheral cities basically serve local enterprises. Most of the industrial platforms are in a fragmented state, lacking of key cross-regional cooperation mechanism.

Construction of transportation networks and cooperation platforms lags behind. The traffic network is also "strong in the east and weak in the west" in the Greater Bay Area. There are seven airports with passenger capacity in the Greater Bay Area, of which Hong Kong, Guangzhou and Shenzhen account for more than $90 \%$ of the total passenger traffic. The efficient and convenient air transport services provided by these airports are mostly concentrated in the eastern part of the Pearl River. Moreover, the lack of efficient inter-city rail connections in the Greater Bay Area limits the integration of the cities in the Greater Bay Area. Similarly, the Hong KongZhuhai-Macao Bridge is constrained by the poor connection of cross-border regulations, which cannot fully meet the capacity demands of the cities. Therefore, the efficient industrial linkage cannot be fully sustained.

\subsection{The industrial competition}

Technological innovation and research capacity are not sufficient. The basic research and development capacity is still insufficient in the region. The upstream market of the new generation information technology industry is monopolized by large MNCs in the United States or Japan (Zhao \& Pei, 2019; Zhang, 2019). [14,15] The local industries face severe risks of "value chain disruption". For example, the cloud computing industry in the Greater Bay Area encounters significant technical barriers. The linkage of the core hardware manufacturing between various cities is weak. Biomedical enterprises have not yet made breakthroughs in the key technologies of the raw material. High-end raw materials such as serum-free medium still rely heavily on imports.

The modern service sector is not mature. The overall service industry in the Greater Bay Area still lags behind the other three Bay Areas in the world. The other three Bay Areas have developed mature service economic forms (Zhang \& Gu, 2017). ${ }^{[16]}$ However, as of 2019, the proportion of the tertiary industry in the Greater Bay Area is about $66.2 \%$, which still lower than that of the San Francisco Bay Area (71.6\%), the New York Bay Area $(82.9 \%)$ and the Tokyo Bay Area (74.5\%). The current industrial structures of Foshan, Dongguan, Huizhou and Zhongshan still need to be improved (Figure 1). According to some research, the service sector could be divided into low-end, mid-end and high-end. The Greater Bay Area is still dominated by low-end and mid-end producer services, while high-end producer services need to be further developed.

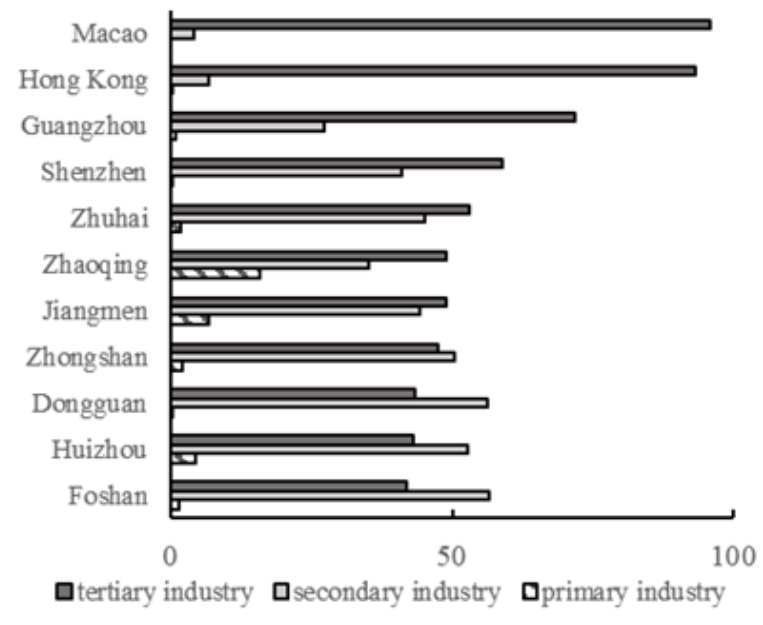

Fig 1. Proportion of three major industries in Greater Bay Area, 2019 


\subsection{International Industrial Cooperation}

The momentum of trade is declining. From 2010 to 2019, the growth rate of foreign trade in the Greater Bay Area declined from $25.85 \%$ to $-5.14 \%$, a significant gap of 31 percentage points (Figure 2). Only Guangzhou, Shenzhen and Foshan have a total trade volume of more than 100 billion yuan, and they account for $78.67 \%$ of the nine cities in the Greater Bay Area. This reflects that the development of foreign trade is highly concentrated in several core cities, and with a huge inter-city gradient. In addition, the Greater Bay Area relying on Hong Kong's trade is not conducive to enhancing the effectiveness of international industrial cooperation.

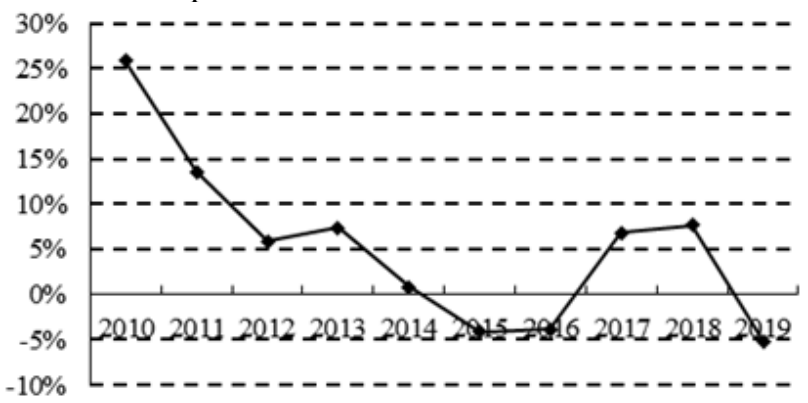

Fig 2. Growth rate of goods trade in Greater Bay Area, 20102019

International investment is overly dependent on Hong Kong. In 2019, the Greater Bay Area's proportion of actual utilization of foreign investment from Europe and the United States to total FDI is less than 2\%, the investment cooperation with developed regions is still insufficient. The relatively single mode of investment and the weakened momentum of international engineering contracting development have gradually come to the fore, which may make it difficult for the Greater Bay Area to capture the new round of global technology transfer and model innovation, or make it neglect the construction of enterprise marketing channels. To a certain extent, the situation of internal consumption of resources appears, and it is difficult to promote the Greater Bay Area and the international level of convergence, and difficult to achieve economic "external circulation".

\section{MEASURING THE INDUSTRIAL POTENTIAL IN THE GREATER BAY AREA CITIES}

\subsection{Methodology}

In this paper, we employ a quantitative method to identify the industrial potentials of the 9 inland cities in the Greater Bay Area. Then we examine the foundations and challenges of the Greater Bay Area's participation in the "dual circulation".

Based on the Product Complexity Index, which is measured by the Harvard Growth Lab using the HS4 code, we construct Industry Complexity Index for 18 manufacturing and 4 service sectors. A 2x2 model matrix was constructed by combining the five-year compound growth rates of the corresponding industries.

\subsection{Visualization of the result}

Figure 3 reveals the visualization result of the industrial development measurement in the Greater Bay Area. We conclude three stylized facts.

First, the center of the industrial distribution locates in the range of high growth and high complexity, indicating that the industrial development of the Greater Bay Area is of high quality and has a solid foundation of regional industrial intra-circulation.

Second, the distribution extends from the lower left to the upper right, showing that the output value of industries with low compound growth rate and low industrial complexity is smaller. While that with high compound growth rate and high industrial complexity is higher, which is in line with the benign development trend of regional economy.

Third, the major industries locate in the positive side of growth rate, and the compound growth rate is mostly maintained in the high growth range of $20 \%$ or even $30 \%$. The electronic equipment manufacturing industry in Shenzhen, Dongguan is maintaining a large scale, but also obtain a compound growth rate of more than $50 \%$. While those locate in the negative growth range are mostly traditional production capacity such as textile and garment manufacturing, which means that the supply-side structural reform has proved to be effective.

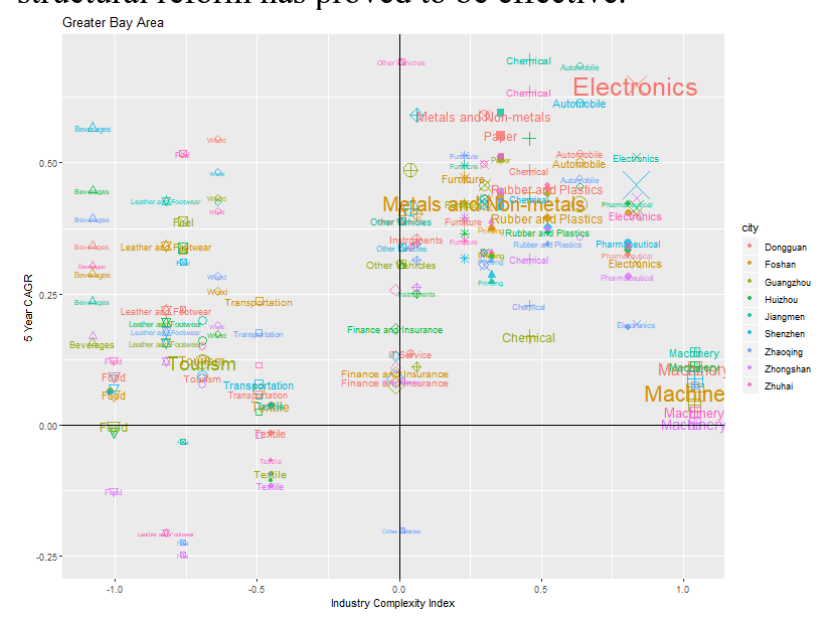

Fig 3. Visualization results of industrial development calculation

a. Different dot shapes represent different industries.

b. For the same industry in all cities, the Industry Complexity Index is the same.

c. The shape and size of fonts and dots represent the size of the output value of the industries.

Based on Figure 3, the following findings could be concluded:

(1) The industries in the Greater Bay Area have a sufficient momentum of development and possess a solid foundation for the "dual circulation".

(2) Regional synergies are better in industries with high complexity and high value-added, such as 
pharmaceutical manufacturing, electronic equipment manufacturing and machinery manufacturing.

(3) The auto manufacturing industry is rapidly forming a pattern of regional synergistic development. Its growth rate in many cities of the Greater Bay Area is extremely high, which is a potential area to promote the construction of "dual circulation".

(4) The development momentum of some advantageous industries is slowing down, especially the machinery manufacturing industry. It is urgent to accelerate the supply-side structural reform and innovation to enable the transformation of the drivers.

\section{POLICY RECOMMENDATIONS}

To further facilitate the Greater Bay Area's participation in constructing the "dual circulation" economy, we propose the following policies.

First, the west bank of the Greater Bay Area should learn from the "German model" and prioritizes machinery, metal and non-metal mineral smelting and processing, and automobile (and other carrier) manufacturing industries. The east should learn from the "California model" and focuses on building an industrial system for the manufacturing of high-tech electronic equipment.

Second, accelerating the high-quality development of pharmaceutical manufacturing and other industries with advantages in the area. It is suggested to build a regional industrial pattern with gradients and priorities, participating in the international economic cycle by relying on advantageous industries while consolidating a solid foundation of industrial intra-circulation.

Third, strengthening the role of Guangzhou as a liaison hub connecting the eastern and western sides, and fully dredge the channels for the flow and circulation of advantageous industrial elements. Enhance Shenzhen's role as a hub connecting ICT service and electronic equipment manufacturing elements on the east and international financial resources in Hong Kong at the south of the Greater Bay Area. Strengthen Shenzhen's ability to integrate international financial, information and technology elements to lead the Greater Bay Area to participate in the "dual circulation".

Last, the cities in the Greater Bay Area should collaborate to play their complementary advantages, and the industrial advantageous areas must strengthen the spatial overflow to make up for the shortcomings of the disadvantaged areas. Accelerate the formation of industrial development synergy and build a solid foundation of the "dual circulation" industrial economic system.

\section{ACKNOWLEDGMENT}

This article was supported by National Social Science Fund in 2020: "Research on Constructing an Internationally-Competitive Modern Industrial System in the Guangdong-Hong Kong-Macao Greater Bay Area" (20\&ZD085), National Natural Science Foundation of China (71603060; 71873041; 72073037) and Guangdong soft science research program (2019a101002100).

Li Qing is the corresponding author, the e-mail is gwliqing@163.com.

\section{REFERENCES}

1. Na Yang. Innovation and Exploration of Global Economic Governance Mechanisms: Taking the Construction of RCEP as an Example. International Economics and Trade Research, 2020,36(12):67-81.

2. Yueqin Lin. Global Economic Governance Reform and the Enhancement of Emerging Countries' Institutional Power of Discourse. Journal of Social Sciences, 2020(11):16-28.

3. Ferrannini A, Barbieri E, Biggeri M, Di Tommaso, MR. Industrial policy for sustainable human development in the post-Covid19 era. World Development, 2021, 137:105+215.

4. Bishop, ML, Payne, A. The political economies of different globalizations: theorizing reglobalization. Globalizations, 2021, 18(1):1-21.

5. Shenrong Shen, Qian Zhao. Promoting High-Quality Economic Development During the 14th Five-year Plan Period by Adopting New Development Pattern of Dual Cycle. Economic Review Journal, 2020(10):18-25.

6. Chaoxun Sheng. Thinking and Countermeasures of Promoting the Conversion of New and Old Kinetic Energy in the 14th Five- Year Plan Period. Reform, 2020(02):5-19.

7. Blanchard J F, Zeng K. China's Role in Global Economic Governance Revisited: Evidence from a Comparative Analysis of Chinese and Japanese BITs. The Chinese Journal of International Politics, 2020, 13(4):553-575

8. Eddie C.M. Hui, Xun Li, Tingting Chen, Wei Lang. Deciphering the spatial structure of China's megacity region: A new bay area--The Guangdong-Hong KongMacao Greater Bay Area in the making, Cities, 2020, 105:102+168.

9. Mei Yan, Jianli Liu. Ideas and Countermeasures for high quality industrial development in GuangdongHong Kong-Macao Greater Bay Area during the 14th Five-Year Plan Period. Enterprise Economy, 2020,39(12):121-130.

10. Yonghui Han, Liangxiong Huang, Xianbin Wang. Do Industrial Policies Promote Industrial Structure Upgrading? Theory and Evidence from China's Development-oriented Local Government. Economic Research Journal, 2017,52(08):33-48.

11. Peiyuan $\mathrm{Xu}$, Guihua Wu. Spatial Evolution of the Knowledge Innovation Network in Guangdong-Hong Kong-Macao Greater Bay Are -- The Role of Shenzhen Technological Innovation Hub. China Soft Science, 2019(05):68-79.

12. Xiangfeng Liu, Qiang Liu. Research on the reform of factor market allocation. Macroeconomic, 
2019(12):34-47+166.

13. Xiang Li, Feng Deng. Technological innovation, industrial structure upgrading and economic growth. Science Research Management, 2019, 40(03):84-93.

14. Yulin Zhao, Chengchen Pei. Technological Innovation, Industrial Convergence and Manufacturing Transformation and Upgrading. Science \& Technology Progress and Policy, 2019, 36(11):70-76.

15. Yan Zhang. Research on the change of international competitiveness of manufacturing industry in China, America and Japan in global value chain system -Based on the perspective of intermediate goods and added value. Inquiry Into Economic Issues, 2019(05):107-118.

16. Rixin Zhang, Zhuotong Gu. The Origin and Next Move of Guangdong-Hong Kong-Macao Greater Bay. Reform, 2017(05): 64-73. 